Sampling bias is an evaluator's worst foe: A research note on an evaluation study of predictors for success in medical school

\author{
Martin Voracek
}

Department of Basic Psychological Research, School of Psychology, University of Vienna, Austria

Martin Voracek, DSc, PhD

Department of Basic Psychological Research

School of Psychology

University of Vienna

Liebiggasse $5 / 3$

A-1010 Vienna, Austria

E-Mail: martin.voracek@univie.ac.at 


\section{Sampling bias is an evaluator's worst foe: A research note on an evaluation study of predictors for success in medical school}

Frischenschlager, Haidinger, and Mitterauer [1] (FHM hereafter) reported an evaluation study of predictors for success in Austrian medical undergraduates. Since most of the international literature on medical education topics still comes from the USA and the UK, in principle this Central European contribution is much needed and to be welcomed. However, several obvious and grave study limitations make the data and findings of FHM markedly less useful than the authors suggest. Relatedly, in the current absence of other Austrian studies on that topic it is foreseeable that the FHM evidence could be used to inform policy decisions in regards to medical education issues in Austria and, possibly so, elsewhere in Central Europe. I strongly opine that, due to the study's design deficiencies, this should be avoided. The main purpose of the present comment is therefore to elucidate the shortcomings of this study as well as to stimulate further discussion and research.

FHM are well aware of the fact that some of their key findings clearly differ from or even contradict previous evidence, but attribute this to differences in study design (their study was a prospective one, whereas many related studies have employed retrospective designs). In contrast, the argument adduced here is that not design differences, but rather design deficiencies, most likely are responsible for the discrepant findings of FHM. In what follows, I will bring forward several lines of evidence--occasionally underpinned with analyses of own, hitherto unpublished data--that all converge in being consistent with this argument. The focus is on five points: firstly and secondly, on deficiencies in study design and in the data analysis; thirdly and fourthly, an inspection and re-evaluation of some key as well as side findings; and fifthly, on other person factors (not considered by FHM) relevant for success in medical school.

Firstly, there are clear deficiencies in study design. FHM mention that for the academic year 2002 to 2003 a total of 1327 freshmen were enrolled at the University of Vienna Medical School. Only 674 (or 50.8\%) students of this cohort completed the survey instrument which, 
crucially so, was administered during lectures for which student attendance apparently was not obligatory. This is a weak procedure for data collection and almost certainly created sampling bias, since it is hard to believe that students were missing completely at random in regards to the variables under scrutiny. Although FHM briefly acknowledge this fact in their discussion section (speaking of a possible "selection bias"), the paper's abstract lacks this important information. Further on, surprisingly so, the authors do not report any test for sampling bias, i.e., whether their self-selected study participants possibly differed significantly from the underlying population of all freshmen, when in fact this is a standard procedure in evaluation research [2]. Regarding the expectable impact of sampling bias in the FHM study, I give just one example. Temporal sampling effects in university subject pools have been described, such that there are differences both in personality and in academic achievement between students who, for course credit, self-select to participate in a study either earlier or later during a term $[3,4]$.

FHM further restricted their sample by choosing to compare two extreme groups only. One group was comprised of 115 "successful" students (those, who passed the SIP exam, i.e., a compulsory achievement test after the first year, at first attempt with grades 1, 2 or 3), whereas the other group was comprised of 130 "unsuccessful" students (those, who failed the SIP exam twice). Together, these two subgroups account for only one-third of the initial sample of FHM and for less than one-fifth of the underlying student population eligible for study participation. It is clear that the explanative power of categorical or continuous predictors is overstated through extreme-group comparisons. In that sense, these circumstances are yet another study design deficiency as well as a less than optimal data-analytic approach (the latter aspect leading over to my second point, below).

I add that the criteria for the two extreme groups, as defined by FHM, are neither guided by theory nor practically stringent and further, that a proper categorization of SIP exam outcomes is not as straightforward and clear cut as their description would suggest. To begin with, students who achieve grade 4 at their first SIP attempt or any grade from 1 to 4 at their second attempt are "successful" students, too: they may proceed with their study without loss of 
time. Then, another peculiarity is given by the fact that there are not too few students who fail the SIP, but actually achieve a higher total score than others who pass this exam.--How can this be? The SIP exam is a five-hour, 230-item, five-category multiple-choice test divided into six parts of differing content, length, and difficulty. SIP items mostly represent knowledge from anatomy, biochemistry, biopsychosocial models of health and disease, genetics, histology, medical propaedeutics and auxiliary sciences, and physiology, with the more difficult subjects (in particular, physiology) given more weight (i.e., more items). The proficiency level was uniformly set at $51 \%$ for all six SIP parts, and students who fail this level in one part have to reattempt this very part only, whereas those who fail in two or more parts have to repeat the entire SIP exam. Finally, the SIP proficiency level ("fail" with less than 51\% of the maximum score) accords with the grading system used throughout Austrian schools and universities. Basically, it is an inverse ordinal scale, such that low grades indicate high achievement $(1=$ very good, 2 = good, 3 = satisfactory, 4 = adequate, and 5 = inadequate or fail). Because of unequallyspaced intervals, such grading scales discriminate only poorly within the lower achievement levels [5]. As a consequence, the student group defined by FHM as "unsuccessful" is much more heterogeneous than their "successful" group.

Secondly, there are clear deficiencies in the data analysis. FHM administered a 67-item questionnaire, covering basic sociodemographic information (including university registration number), secondary-school performance, living conditions and social integration, study motivation and learning capacity, health and stress coping, and information access of medical freshmen. Most of these items must be regarded as poorly operationalized single-item measures of unknown reliability and validity (e.g., visual analogue scales and self-reported study effort, learning capacity, and learning styles, queried at the very beginning of medical education).

Across tables 1 to 4 of FHM, I count $95 P$ values from bivariate statistical tests, of which 23 are significant $(P<0.05)$. Neither did FHM conduct a multivariate statistical analysis to single out the truly salient predictors for student success from the bulk of their variables, nor did the authors try to statistically arrive at more reliable and sound aggregate measures, nor was there 
an attempt to control for the inevitable increase in the probability of statistical type 1 errors, due the multitude of significance tests conducted in the study. These are important shortcomings, since quite a number of predictors are highly intercorrelated (e.g., four secondary-school marks plus their total [5], or living situation, social, and economic variables that jointly originate in the freshmen's family background). It is both clear that several findings of FHM simply must be type 1 errors that will not be replicable in future research and that most of their significant findings would not survive usually applied controls for the inflation of type 1 error probability (the Bonferroni-corrected significance level would be at $0.05 / 95=0.000526$ ).

Thirdly, inspection of some key findings of FHM shows them at odds with established knowledge in this research area. For instance, FHM found female students to be overrepresented in the "unsuccessful" group. Since females constitute about two-thirds of all Austrian medical students, the study failure of the largest demographic subgroup within this population would be a worrying state of affairs. Although the authoritative work of Ferguson and colleagues [6] is cited (ref. 5 of FHM), which is a systematic, up to date review of the world literature on success factors in medical school, FHM do not mention that this review concluded (based on 16 papers) that in fact female, not male sex is associated with success in medical training.

Likewise, FHM found students with mother tongue other than German to be overrepresented in the "unsuccessful" group. Current evidence from the international literature pertaining to the success rate of ethnic minority members in medical training is quite mixed (based on 14 papers, reviewed by Ferguson and associates [6]). Importantly so, one recent study [7] found that ethnic minority students not born in the UK performed better than white UK students, whereas UK-born ethnic minority students performed worse. It is both evident that the ethnic minority definition used by FHM does not unequivocally differentiate between ethnic minority students born in Austria and those born abroad and that the evidence of FHM on the study success of this student group is based on merely 32 subjects at most (calculated from table 1 of FHM), given that there were no missing values for this variable (which seems unlikely, since there were already 12 missing values across 245 participants for the participant sex variable). 
FHM (last sentence of their discussion section) invite replications of their study involving other university studies, and I have followed their lead. Here, unpublished data on sex differences in grades on a mandatory elementary course in statistics and research methods for first-term psychology students are presented. The data are from the University of Vienna School of Psychology, where the majority of Austrian psychologists enroll and graduate. Notice that the sample size (1658, 82.4\% female) is larger by a factor of almost 7 than the sample size of FHM, that these data are population-based (i.e., representing the entire cohorts of the three most recent academic years), and that there is abundant evidence for sex differences in higher, but not elementary, mathematical abilities, favoring males [8]. Like FHM (see their table 1), I took the liberty to apply a parametric approach in analyzing ordinal-scaled grades. Briefly, according to this data set, there are nil sex differences in the elementary statistics course grades, $M(S D)$ and fail rates being 2.18 (1.37) and 9.9\% for males and 2.11 (1.28) and 7.3\% for females, with students' sex accounting only for a minute and thus negligible proportion of the variance (less than $0.1 \%$ ) in the course grades. In all, I think it is more parsimonious to assume that key findings of FHM are due to factors unique to their sample (sampling bias and type 1 errors) than to ascribe them veracity.

Fourthly, inspection of some supplemental findings of FHM also shows them at odds with either findings from related research or common knowledge or both. To anticipate my conclusion: it is the same as in the above section. It is indeed hard to believe, if not counterintuitive, that, according to FHM, "successful" medical students should have thought more often about studying something else than medicine and should have had the wish to study medicine for a shorter period of time than the "unsuccessful" ones. Discussing these unusual findings, FHM speculate that such features indicate a more mature and instrinsic study motivation. One should not indiscriminately buy a post hoc explanation such as this one. A more plausible idea appears to be that these anomalous findings could have something to do with the poor operationalization and instrumentation of the constructs FHM intended to measure (see section one, above). 
Another side finding of FHM is that students who enrolled later (as judged from the within-year substring of their university registration number) were overrepresented in the "unsuccessful" group. Discussing this "late-comer" effect, FHM conjectured that it could be a pointer to poor personal organization and study motivation. Using once again the statistics course data (analyzed in section three, above), I was interested whether the "late-comer" effect would replicate in another sample.--It did not: there was no association between registration number and statistics course grade (Pearson's correlation coefficient: -0.012 , Kendall's rankorder correlation coefficient: 0.027 ; both are far away from statistical significance even with a sample size of 1658 subjects available for this analysis).

Fifthly, FHM did not consider other person factors that are relevant for success in medical school. This final section of the present comment is intended to indicate some thoughtstimulating and useful avenues for future research in this area. I give two examples, the first one demonstrating the salience of personality factors. In a recent prospective cohort study from the UK [2] (cited by FHM, their ref. 6), the Big-Five personality dimension of conscientiousness consistently turned out to be the best predictor for student performance over the five years toward the medical degree. To explain, individuals high in conscientiousness are careful, hardworking, organized, practical, and thorough in what they do, and, unsurprisingly so, this personality factor is known to be a significant general predictor of job performance and trainability.

The other example concerns the well-known fact that attending medical school is strongly familial [9]. One study of Huckle and McGuffin in the UK [10] overall found about $13.5 \%$ physicians among medical students' first-degree relatives (parents and siblings). Since physicians amount only to $0.2 \%$ of the general population in the UK, the "relative risk" for studying medicine, given a physician among ones first-degree relatives, is about 65 , which must be viewed as an exceedingly potent "risk factor". Interestingly so, it appears that this familiality pattern is specific for medicine, since in the same study the corresponding figure for zoology students was about $5 \%$ only. 
Unpublished data of my own, which bear further on this issue, are presented here. The data set (356 final-year medical students, 56.5\% female) was collected during the academic year 2000 to 2001 at the University of Vienna Medical School. The sample is a true $50 \%$ random sample, with a response rate of next to $100 \%$, of the entire cohort of final-year students of that institution at that time (for details, see a predecessor study [11]), and almost all of these students must have graduated by now. Among various other variables, information on physicians among first-degree relatives was secured in this student cohort during mandatory small-group courses on psychotherapeutic propraedeutics.

I have the following results: $25.7 \%$ of the "future doctors" (i.e., final-year students) had at least one physician among their first-degree relatives. This finding comes with a substantial as well as statistically significant sex difference (33.1\% of male versus $20.0 \%$ of female students, two-tailed $P=0.005)$. Since physicians amount to about $0.4 \%$ of the general population in Austria, almost the same "risk factor" than in the Huckle and McGuffin study [10] is attained, namely, a relative risk of about 66 in the present data, thus nicely replicating the previous evidence from the UK.

What is of great interest in the context discussed here is the, to my knowledge, novel finding that among "future doctors", males with first-degree relatives in the medical profession were significantly younger (two-tailed $P=0.006$ ) than males without such relatives, $M(S D$ ) for age 25.2 (2.2) and 26.7 (3.4) years, respectively. In the same data set, there was no such pattern for female students. Currently, there are no data available to pinpoint the likely causes for this noteworthy finding. It goes without saying, however, that a mean age difference of one and a half years among final-year medical students of the same sex, depending on whether they have physicians as relatives or not, definitely is not a minor effect. I think it is hard to escape the conclusion that the former student group must have studied more efficiently, quickly, and successfully than the latter one.

These two examples of person factors for success in medical school presented here suggest that a number of well-defined and potent success predictors exist over and above those 
studied by FHM. On the other hand, we are all well-advised to practice some modesty in these matters, since evaluation studies of medical education have repeatedly shown that undergraduate achievement is only weakly related to later postgraduate clinical competence [6]. Notwithstanding that, a feature recurrently encountered here is the necessity of investigating unbiased samples or, even better so, entire student cohorts. This closes the chain of argumentation, by leading back to my first point of criticism. Hence let me conclude allusively, with a wordplay: while some have asserted that diamonds are a girl's best friend or that gentlemen prefer blondes--which, of course, is debatable--it is without a doubt that sampling bias is an evaluator's worst foe and that epidemiologists prefer either random samples or population-based data--that, at least, is for sure. 


\section{References}

1 Frischenschlager 0, Haidinger G, Mitterauer L. Factors associated with academic success at Vienna Medical School: prospective survey. Croat Med J. 2005;46:58-65.

2 Ferguson E, James D, O'Hehir F, Sanders A. Pilot study of the roles of personality, references, and personal statements in relation to performance over the five years of a medical degree. BMJ. 2003;326:429-32.

3 Bernard L. Variations in subject pool as a function of earlier or later participation. Psychol Rep. 2000;86:659-68.

4 Bernard LC, Walsh RP. Variations in a university subject pool as a function of earlier or later participation and self-report: a replication and extension. Psychol Rep. 2002;91:553-70.

5 Lienert GA. Points and grades evaluation [in German]. Frankfurt/M.: Athenäum; 1987.

6 Ferguson E, James D, Madeley L. Factors associated with success in medical school: systematic review of the literature. BMJ. 2002;324:952-7.

7 McManus IC, Richards P, Winder BC, Sproston KA. Final examination performance of medical students from ethnic minorities. Med Educ. 1996;30:195-200.

8 Geary DC. Sexual selection and sex differences in mathematical abilities. Behav Brain Sci. $1996 ; 19: 229-84$.

9 McGuffin P, Huckle P. Simulation of Mendelism revisited: the recessive gene for attending medical school. Am J Med Genet. 1990;46:994-9.

10 Huckle P, McGuffin P. Familial factors in going to medical school. Med Educ. 1991;25:135.

11 Voracek M, Jandl-Jager E, Springer-Kremser M. Medical students' attitudes towards psychotherapay: an intervention-based pre-post comparison study. Wien Klin Wochenschr. 2001;113:408-15. 